\title{
Gas proportional scintillation counter pulse-signature analysis using digital techniques
}

\author{
P.C.P.S. Simões ${ }^{a, b, *}$ J.M.F. dos Santos ${ }^{a}$, C.A.N. Conde ${ }^{a}$

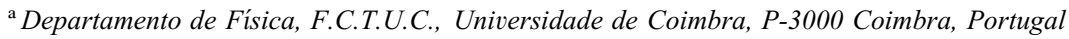 \\ ${ }^{\mathrm{b}}$ Departamento de Biofísica e Biomatemática, Faculdade de Medicina, Universidade de Coimbra, P-3000 Coimbra, Portugal
}

\begin{abstract}
The use of short shaping time-constants $(50 \mathrm{~ns})$ followed by digital sampling of gas proportional scintillation counter pulses is investigated. Such short shaping time causes each pulse to closely resemble the scintillation light burst, allowing for an effective pulse-signature analysis. Pulse amplitude is obtained by numerical integration of the digital pulse samples. The shorter total pulse durations, lead to pulse-height distributions that are much less dependent on the total interaction rate occurring in the detector. Total interaction rates up to $12 \mathrm{k} \mathrm{s}^{-1}$ can be tolerated without significant degradation of the obtained pulse-height distribution. It was also verified that the maximum throughput achieved by the digital pulse-height analyser improves from 1 to $1.7 \mathrm{k} \mathrm{s}^{-1}$ when using the short shaping time-constants. (C) 1999 Elsevier Science B.V. All rights reserved.
\end{abstract}

\section{Introduction}

Gas proportional scintillation counters (GPSC) have been widely applied in important areas such as X-ray astronomy and energy dispersive X-ray fluorescence analysis [1-3]. They combine room temperature operation with large detection area and high counting rates without space charge effects [4-6]. The interest in GPSCs has led to a continued research effort in understanding its working principles and in improving its performance.

Digital signal processing developments have been mostly oriented to nuclear spectrometry for solid-state detector applications. However, the ap-

\footnotetext{
* Correspondence address: Departamento de Física, F.C.T.U.C., Universidade de Coimbra, P-3000 Coimbra, Portugal.
}

plication of these techniques to the analysis of GPSC pulses proved to be a simple but effective method to improve the detector performance in $\mathrm{X}$-ray spectrometry applications, through background reduction and peak enhancement. Recent studies investigated the applications of digital pulse processing techniques to X-ray spectrometry with GPSCs [7-9] using a digital pulse-height analyser (DPHA).

In GPSCs applications long pulse shape timeconstants are routinely used (typically $5 \mu$ s for both integration and differentiation time-constants) for best performance. Pulse integration was performed directly by the main amplifier $[7,8]$ or else digitally by the DPHA [9]. X-ray pulse-height distributions were improved on the basis of digital pulse risetime discrimination [7-9]. 
In the present work we investigate the advantages of using very short time-constants $(50 \mathrm{~ns})$ in the main amplifier. Because of the short time-constants the pulse shape will closely resemble the scintillation light burst. This allows pulse-signature identification, an advantage in applications where pulse shape discrimination of multiple events is needed $[10,11]$. On the other hand, since the total pulse duration is shorter, the obtained pulse-height distributions will be much less dependent on the interaction rates. This will present some advantages in GPSC applications where high interaction rates are involved, such as in X-ray solar flares, synchrotron radiation and X-ray structure analysis $[3,6]$.

\section{Results and discussion}

\subsection{Experimental set-up}

The schematic diagram of the uniform field GPSC used in this work is presented in Fig. 1. The detector has a $4 \mathrm{~cm}$ deep absorption region and a $1 \mathrm{~cm}$ deep scintillation region and it is described in detail in Ref. [12].

The GPSC signals are fed to the DPHA through a HP5554A preamplifier and a HP5582A main amplifier using $50 \mathrm{~ns}$ integration and differentiation time-constants. The short differentiation time constant causes the pulse shape to follow the scintillation light burst, while the short integration timeconstant eliminates high-frequency noise. Each pulse is digitised at a $20 \mathrm{MHz}$ rate with 8-bit resolution and processed by the DPHA [7].

In a typical run, each pulse is processed by a series of algorithms as follows. The digitised pulse samples are smoothed by a 3-point median filter to reduce high-frequency oscillation [13]. The pulse amplitude is obtained by numerical integration of the pulse samples. Pulse duration is taken as the time that the pulse spends above a threshold value set just above noise level. A pulse is rejected whenever a sampled value overshoots the ADC scale.

Pulse discrimination is now performed on the basis of pulse duration. As previously [7], partial pulse-height distributions discriminated according

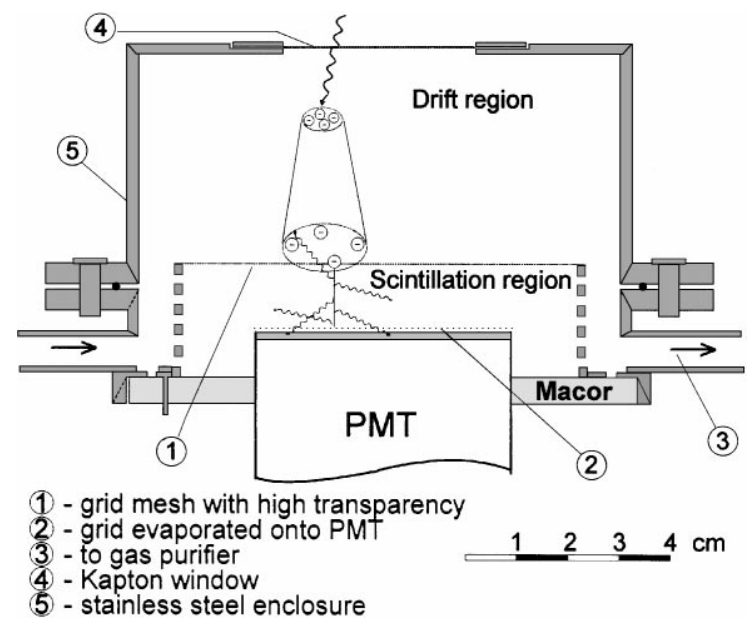

Fig. 1. Schematic diagram of the GPSC used in this work.

to different pulse duration can be simultaneously acquired.

\subsection{Pulse-signature analysis}

A non-collimated ${ }^{109} \mathrm{Cd}$ radioactive source was placed in the active volume of the GPSC filled with pure xenon at $1330 \mathrm{mbar}$. Each ${ }^{109} \mathrm{Cd}$ decay may involve the ejection of a conversion electron (CE) $\left(e_{\mathrm{K}}=62.5 \mathrm{keV}\right.$ or $\left.e_{\mathrm{L}}=84.6 \mathrm{keV}\right)$ accompanied by the simultaneous emission of an $\mathrm{Ag}$ fluorescence X-ray photon $\left(K_{\alpha}=22.1 \mathrm{keV}, \quad K_{\beta}=25.0 \mathrm{keV}\right.$, $L_{\alpha}=3.0 \mathrm{keV}, L_{\beta}=3.3 \mathrm{keV}$ ) or Auger electrons.

In Fig. 2 are presented a set of digitised pulses. Only pulses with an area corresponding to $22.1 \mathrm{keV}$ $\left(\mathrm{Ag}-\mathrm{K}_{\alpha} \mathrm{X}\right.$-rays or background events) are shown. As seen, different pulse signatures can be distinguished. Pulses $a$ result from events occurring in the drift region near the detector axis and leading to a single primary electron cloud. Pulses $b$ result from events where the electron cloud due to the Xe photoelectron emission and the electron cloud due to Xe L-fluorescence secondary emission reach the scintillation region at different instants. Pulses $c$ result from events where the primary electron cloud is strongly distorted, e.g. events occurring near detector walls. Note that the number of events $a$, $b$ and $c$ presented in Fig. 2 are not normalised to their occurring probability but the number of events $a$ is largely dominant. 


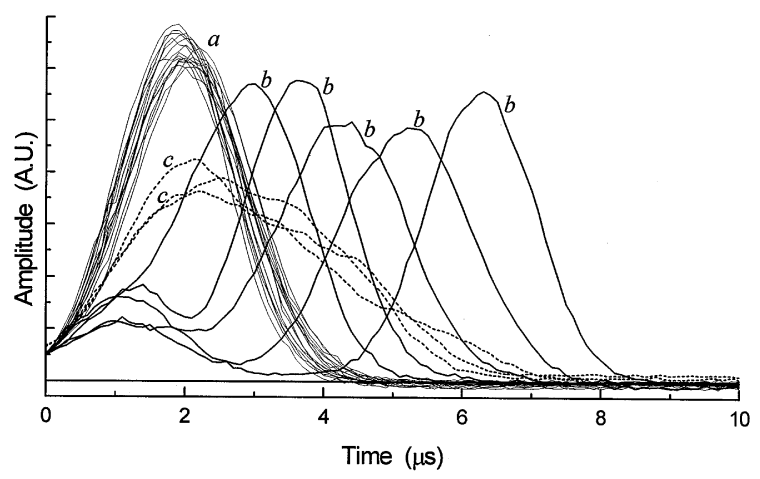

Fig. 2. Digitised pulses, from a ${ }^{109} \mathrm{Cd} \mathrm{X}$-ray source, with an area correspondent to $22.1 \mathrm{keV}$. Pulses $a$ result from events occurring in the drift region and leading to a single scintillation light burst. Pulses $b$ result from events where the scintillation light burst from Xe L-fluorescence secondary emission is identifiable. Pulses $c$ result from events where the primary electron cloud is strongly distorted.

Fig. 2 shows that the method in study is an effective and versatile technique to discriminate events that produce a characteristic pulse signature, e.g. events leading to double interaction points like pulses $b$ in Fig. 2. In fact this is the working principle of a gated GPSC $[10,14]$.

In Fig. 3, curve $a$, represents the pulse-height distribution obtained from the non-collimated ${ }^{109} \mathrm{Cd}$ radioactive source. The real count rate is about $2 \mathrm{k} \mathrm{s}^{-1}$. The Ag K-fluorescence lines and the $e_{\mathrm{K}}$ and $e_{\mathrm{L}} \mathrm{CE}$ peaks can be distinguished. The low-energy tail associated to each peak results from solid angle effects and primary electron loss to the detector walls, since the radioactive source is not collimated. Using pulse-duration discrimination the background under the $22.1 \mathrm{keV} \mathrm{X}$-ray line is efficiently reduced as in previous work [7-9]: curve $b$ of Fig. 3 is the pulse-height distribution obtained when only the pulses with $3.6-4.0 \mu$ s pulse duration time are counted.

The $e_{\mathrm{K}}=62.5 \mathrm{keV}$ CE emission occurs simultaneously either with a $22.1 \mathrm{keV}$ fluorescence emission $\left(83 \%\right.$ of $e_{\mathrm{K}}$ events) or with Auger electrons $\left(17 \%\right.$ of $e_{\mathrm{K}}$ events). Thus, this allows an unique identification of the $62.5 \mathrm{keV} \mathrm{CE}$, since a unique pulse signature is produced whenever the electron clouds of the $22.1 \mathrm{keV}$ fluorescence emission and of the $62.5 \mathrm{keV} \mathrm{CE}$ reach the scintillation region at

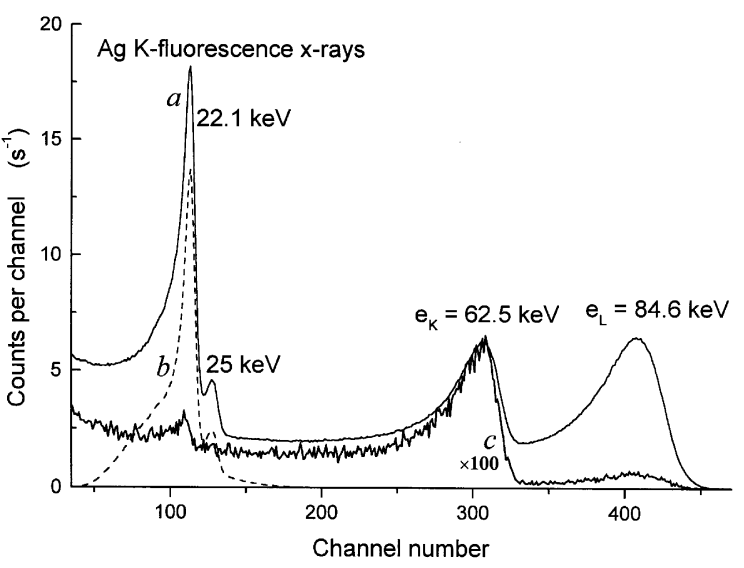

Fig. 3. Pulse-height distribution obtained from a non-collimated ${ }^{109} \mathrm{Cd}$ radioactive source placed inside the detector active volume. Curve $a$ shows the distribution obtained without pulse-duration discrimination. Curve $b$ is obtained after pulseduration discrimination in the range from 3.6 to $4 \mu \mathrm{s}$. To the pulse-height distribution shown in curve $c$ contribute only the pulse that appears within a $20 \mu$ s time interval after a triggerpulse with area correspondent to $22.1 \mathrm{keV}$ with time-duration in the range $3.6-4 \mu \mathrm{s}$. Curve $c$ is represented with a vertical scale multiplied by a factor of 100 .

different instants. When the electron clouds of both events reach the scintillation region simultaneously a single pulse results. In this case, the pulse cannot be distinguished from the $84.6 \mathrm{keV}$ CE pulses.

Curve $c$ in Fig. 3 represents the pulse-height distribution obtained when only the first pulse following a trigger pulse, within a $20 \mu$ s time-window interval, is considered. The pulses selected for triggering are those with an area corresponding to $22.1 \mathrm{keV}$ and a 3.6 to $4.0 \mu$ s time-duration. As it is shown the $62.5 \mathrm{keV} \mathrm{CE}$ is the only prominent feature on the pulse-height distribution. Its energy resolution and peak-to-background ratio improves relatively to the non-triggered pulse-height distribution from $11.6 \%$ to $9.7 \%$ and from 2 to 6 , respectively, thus improving the detector sensitivity to the $62.5 \mathrm{keV}$ CE. The detection efficiency is reduced by about two orders of magnitude, though, due mainly to the $\mathrm{Ag} \mathrm{K}$-fluorescence yield, to the $\mathrm{Ag} \mathrm{K}_{\alpha} / \mathrm{K}_{\beta}$ relative emission probability, to the detector $a b$ sorption efficiency of the $22.1 \mathrm{keV} \mathrm{X-rays} \mathrm{and} \mathrm{to} \mathrm{the}$ probability of the electron clouds of $\mathrm{CE}$ and $\mathrm{K}_{\alpha}$ events lead to a single pulse. 


\subsection{Performance at high count rates}

Another advantage of using very short time-constants in the main amplifier is the less dependence of the pulse-height distribution on the total interaction rate, since pulse duration is shorter.

Pulse-height distributions were obtained from $2 \mathrm{~mm}$ collimated ${ }^{55} \mathrm{Fe},{ }^{109} \mathrm{Cd}$ and ${ }^{241} \mathrm{Am} \mathrm{X}$-ray sources, directly irradiating the GPSC, and for total rates from $10^{2}$ to $3 \times 10^{4}$ interactions per second. In Fig. 4 curves $a, b$ and $c$ depict the pulse-height distributions obtained from a ${ }^{109} \mathrm{Cd}$ $\mathrm{X}$-ray source irradiating the detector at 0.1 , 12 and $18 \mathrm{k}$ total counts per second, respectively. The pulse-height distributions in Fig. 4.1 are obtained with a standard Wilkinson-type pulse-height analyser (PHA) (Nucleus-PCAII), while the distributions in Fig. 4.2 are obtained with the DPHA. The shaping time-constants used for the analog PHA acquisitions are selected to obtain the best performance for each count rate: 5, 2 and $2 \mu$ s, respectively.

In Fig. 5 we present the energy resolution as a function of the real count rate for 5.9, 22.1 and $59.6 \mathrm{keV} \mathrm{X}$-rays, as measured on the pulse-height distributions obtained with the Wilkinson-type PHA and with the DPHA. A pulse-duration discrimination was selected in the DPHA so that a counting-peak efficiency of more than $90 \%$ is maintained. As it can be seen, the energy resolution

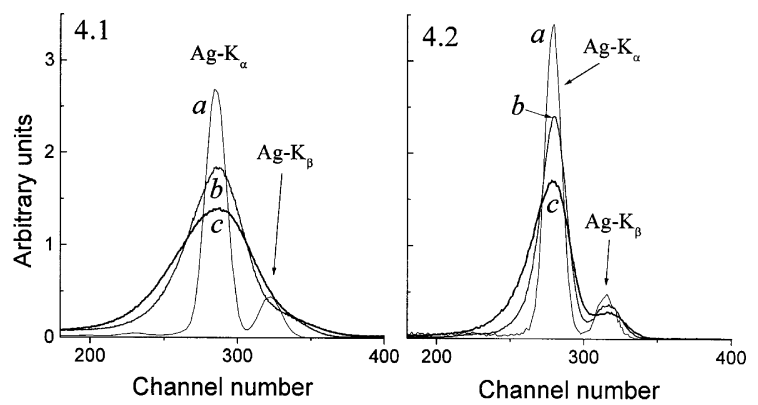

Fig. 4. Pulse-height distributions obtained from a ${ }^{109} \mathrm{Cd} \mathrm{X}$-ray source irradiating the detector at $0.1,12$ and $18 \mathrm{k}$ total counts per second, curves $a, b$ and $c$, respectively: (4.1) using a standard Wilkinson-type PHA; (4.2) using the DPHA. The area under each curve is normalised. obtained using the DPHA is less dependent on the count rate than the resolution obtained using the Wilkinson-type PHA. Since at high count rates the best shaping time-constant used with the Wilkinson-type PHA decreases with decreasing X-ray energy (e.g. $0.5 \mu$ s shaping time-constants for the ${ }^{55} \mathrm{Fe}$ at $30 \mathrm{k}$ counts per second), the difference in the performance shown for both PHAs is smaller for low X-ray energy, as shown in Fig. 5. One of the reasons for the versatility of the DPHA technique is the ability to perform pulse analysis using always the same short shaping time-constant while for the Wilkinson-type PHA one must select the best shaping time-constants according to the total count rate and the X-ray energy.

The performance obtained with the DPHA operating in the earlier conditions [7-9] presents the same dependence with the count rate as that obtained with the Wilkinson-type PHA.

In Fig. 6 we depict the pulse-height distributions obtained from lead $(\sim 100 \mathrm{ppm})$ in a water sample excited with a ${ }^{109} \mathrm{Cd} \mathrm{X}$-ray source. The total count rate is about $18 \mathrm{ks}^{-1}$. An energy resolution and a peak-to-background ratio for the $\mathrm{Pb}-\mathrm{L}_{\beta}$ line $(12.6 \mathrm{keV})$ of $9.3 \%$ and 0.29 are obtained when using the Wilkinson-type PHA and shaping timeconstants of $2 \mu \mathrm{s}$, curve $a$, while the values $7.9 \%$ and 1.7 are achieved when using the DPHA, curve $b$. As before, a pulse-duration discrimination was selected in the DPHA so that a counting peak

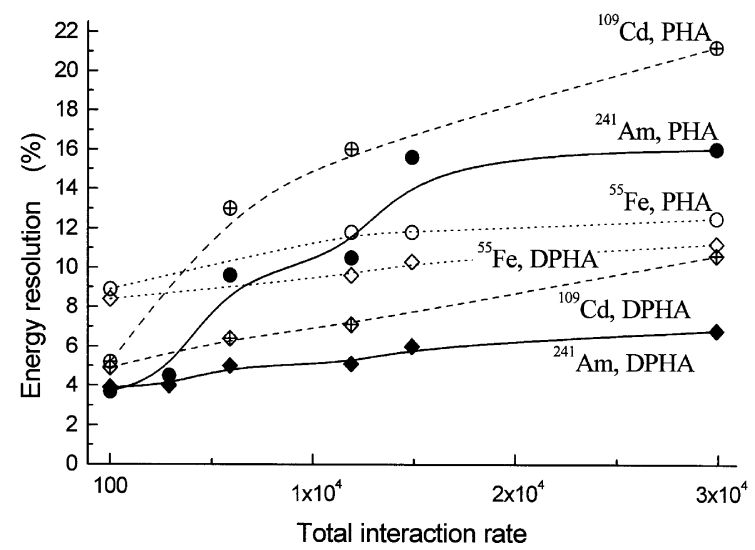

Fig. 5. Energy resolution as a function of the real count rate for 5.9, 22.1 and $59.6 \mathrm{keV} \mathrm{X}$-rays, as measured for the pulse-height distributions obtained with the Wilkinson-type PHA and with the DPHA. 


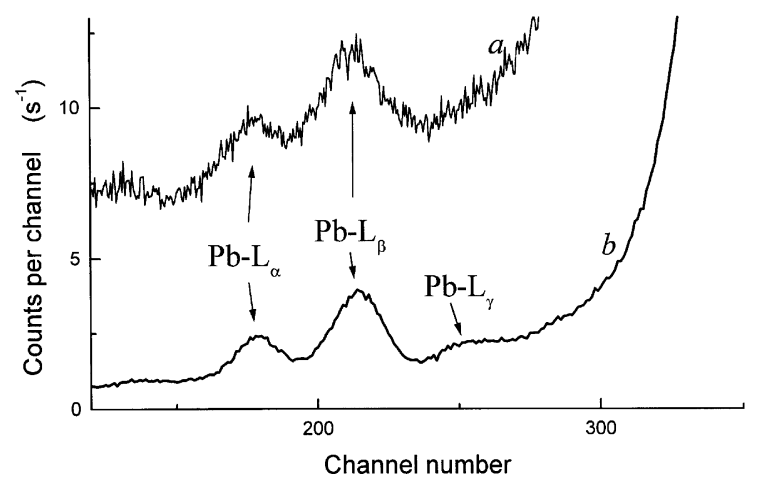

Fig. 6. Pulse-height distributions obtained from lead $(\sim 100 \mathrm{ppm})$ in a water sample excited with a ${ }^{109} \mathrm{Cd}$ source for a total count rate of about $18 \mathrm{k} \mathrm{s}^{-1}$, obtained with a standard Wilkinson-type PHA, curve $a$, and obtained with the DPHA, curve $b$.

efficiency of more than $90 \%$ is maintained. For a total count rate of about $150 \mathrm{~s}^{-1}$, the energy resolution is $7.8 \%$ and $7.1 \%$ and the peak-to-background ratio is 0.78 and 1.7 for the Wilkinson-type PHA and the DPHA, respectively.

\section{Conclusions}

We have demonstrated that the use of short shaping time-constants allows an effective pulsesignature analysis. Since the total pulse duration is shorter, the obtained pulse-height distribution will also be much less dependent on the total interaction rate occurring in the detector. Total interaction rates up to $12 \mathrm{k} \mathrm{s}^{-1}$ can be tolerated without significant degradation of the obtained pulseheight distribution.

Pulse duration and its undershoot are the limiting factors in high count-rate applications. The use of pole-zero cancellation and base-line restorer techniques reduces the effect caused by the pulse undershoot. However, this effect can be simply and likely more efficiently corrected by digital subtraction of the pulse base line.

We have also found that pulse discrimination based on total pulse duration-time is as effective as the previous pulse rise-time discrimination technique $[7-9,15]$.
The peak-to-background ratio is always better when using the DPHA than using the Wilkinsontype PHA $[7-9,15]$, but this difference becomes smaller as the count rate increases.

A slight degradation of the performance obtained using this method compared to other techniques was observed at low count rates, in cases where the noise is not efficiently eliminated by the electronics (e.g. when $\mathrm{HV}$-supply ripple is significant).

The maximum throughput achieved by the DPHA operating with short shaping time-constants improves from 1 to $1.7 \mathrm{k}$ counts per second when compared to the throughput achieved by the DPHA operated in previous configurations. This improvement results from the fact that the number of digitised samples, per pulse, used by the processing algorithms is smaller. The maximum throughput achieved by the DPHA can be significantly increased using an upgraded digital signal processor and faster data transfer (e.g. using direct memory access).

\section{Acknowledgements}

This work was carried out in the Atomic and Nuclear Instrumentation Group of the Instrumentation Centre (Unit 217/94) of the Physics Department, University of Coimbra. This work was supported by CERN/P/FIS/1166/97. Travel support is acknowledged from Fundação Calouste Gulbenkian, Lisboa. The authors wish to thank T.H.V.T. Dias for manuscript reviewing.

\section{References}

[1] A. Peacock, R.D. Andresen, E.A. Leimann, A.E. Long, G. Manzo, B.G. Taylor, Nucl. Instr. and Meth. 169 (1980) 613.

[2] A. Smith, A. Peacock, T.Z. Kowalski, IEEE Trans. Nucl. Sci. NS-34 (1987) 57.

[3] D.A. Goganov, A.A. Schultz, Nucl. Instr. and Meth. 394 (1997) 151.

[4] A.J.P.L. Policarpo, Space Sci. Instr. 3 (1977) 77.

[5] V.P. Varvaritsa, I.V. Vikulov, V.V. Ivashov, M.A. Panov, V.I. Filatov, K.I. Shchekin, Instr. Exp. Tech. 35 (1992) 745.

[6] A. Smith, M. Bavdaz, Rev. Sci. Instr. 63 (1992) 689. 
[7] P.C.P.S. Simões, J.F.C.A. Veloso, J.M.F. dos Santos, C.A.N. Conde, IEEE Trans. Nucl. Sci. NS-44 (1997) 521.

[8] P.C.P.S. Simões, J.M.F. dos Santos, C.A.N. Conde, X-Ray Spectrom. 26 (1997) 182.

[9] P.C.P.S. Simões, J.M.F. dos Santos, C.A.N. Conde, IEEE Trans. Nucl. Sci. NS-45 (1998), accepted for publication.

[10] G. Manzo, J. Davelaar, A. Peacock, R.D. Andresen, B.G. Taylor, Nucl. Instr. and Meth. 177 (1980) 595.

[11] V. Dangendorf, H. Schmidt-Bocking, Proc. SPIE-EUV, $\mathrm{X}$-Ray, and Gamma-Ray Instrumentation for Astronomy
V, 6-11 August 1989, San Diego, California, vol. 1159, 1989, p. 192.

[12] J.M.F. dos Santos, J.F.C.A. Veloso, R.E. Morgado, C.A.N. Conde, Nucl. Instr. and Meth. 353 (1994) 195.

[13] E. Cosulich, F. Gatti, Nucl. Instr. and Meth. 321 (1992) 211.

[14] M.R. Sims, G. Manzo, A. Peacock, B.G. Taylor, Nucl. Instr. and Meth. 211 (1983) 499.

[15] P.C.P.S. Simões, J.C. Martins, C.M.B.A. Correia, IEEE Trans. Nucl. Sci. NS-43 (1996) 1804. 